\title{
Work-in-Progress: Reconfigurable accelerator on FPGA for scientific computing
}

\author{
From a space-borne instrument to a high-performance computing data center \\ J.P. Cobos Carrascosa, D. Hernández Expósito, J.L. Ramos Mas, B. Aparicio del Moral, A. Sánchez \\ Gómez, M. Balaguer, A.C. López Jiménez, D. Orozco Suárez, J.C. del Toro Iniesta \\ Instituto de Astrofísica de Andalucía, IAA-CSIC \\ Granada, Spain \\ \{jpedro,dhdez,ramos,bea,asgomez,balaguer,antonio,orozco,jti\}@iaa.es
}

\begin{abstract}
We present a scientific computing accelerator on FPGA that uses hundreds of processors working in parallel organized in several SIMD cores. The accelerator is installed within an Ethernet network and acts as a high-performance computing server. A prototype is presented for processing solar images and achieves a great performance that can compete with a cluster.
\end{abstract}

\section{KEYWORDS}

FPGA, accelerator, SIMD architecture

\section{FPGA-based accelerator}

The hardware-acceleration through FPGAs is frequently used for achieving embedded high-performance computing (HPC) for astrophysics instruments. They provide plenty of efficient computing resources and high configurability to fulfill the stringent requirements of scientific algorithms. Specifically, it was demonstrated that FPGAs offer enough floating-point capabilities and enable allocation of specific-domain processors to solve high demanding scientific problems [1]. FPGA devices have been used for carrying out complex scientific algorithms, as the inversion of the radiative transfer equation for polarized light (RTE) inversion and a given image compression, aboard the Solar Orbiter's PHI (SO/PHI; PHI is an acronym for Polarimetric and Helioseismic Imager) space instrument. The multi-core architecture on FPGA reached high performance computing by using a relatively slow and old-fashioned FPGA device - Xilinx XQR4VSX55- and under strict time and power constraints [1].

Specifically, the proposal is focused in exploiting data parallelism using several processors working together and using different data input streams. This is using the well- known Single Instruction Permission to make digital or hard copies of all or part of this work for personal or classroom use is granted without fee provided that copies are not made or distributed for profit or commercial advantage and that copies bear this notice and the full citation on the first page. Copyrights for components of this work owned by others than the author(s) must be honored. Abstracting with credit is permitted. To copy otherwise, or republish, to post on servers or to redistribute to lists, requires prior specific permission and/or a fee. Request permissions from Permissions@acm.org.

CASES'19 Companion, October 13-18, 2019, New York, NY, USA

(C) 2019 Copyright is held by the owner/author(s). Publication rights licensed to ACM.

ACM 978-1-4503-6925-1/19/10 ..\$15.00

https://doi.org/10.1145/3349569.3351541 stream, Multiple Data stream (SIMD) processing strategy. That is often very suitable to deal with scientific algorithms that can process in parallel several great tons of data. The final system based on FPGA for SOPHI improves the time and power consumption of ground-based systems based on commercial CPUs, as demonstrated in [1]. This is the seed of the idea for using an FPGA to carry out the processing architecture for other scientific computing problems. Additionally, the FPGA technology has evolved quickly and the internal resources have increased significantly in the last years. So, we propose in this paper a new FPGA-based accelerator that gathers dozens of the SIMD-based cores used in SOPHI. However, there are other elements to provide them with communication and order. The updated architecture is outlined in Figure 1 where each SIMD icore represents the original one. Each SIMD core has the ability of saving resources through allocating operation cores in a shared operation block (SOB), which is accessed by every processor. For example, there is only one floating-point division core because it demands many FPGA resources. It is important to remark that the SIMD cores can be configured to include up to $\mathrm{P}$ processors with only one SOB. This will give great flexibility when the system is programmed for carrying out a specific algorithm and can adapt the architecture to squeeze the FPGA resources.

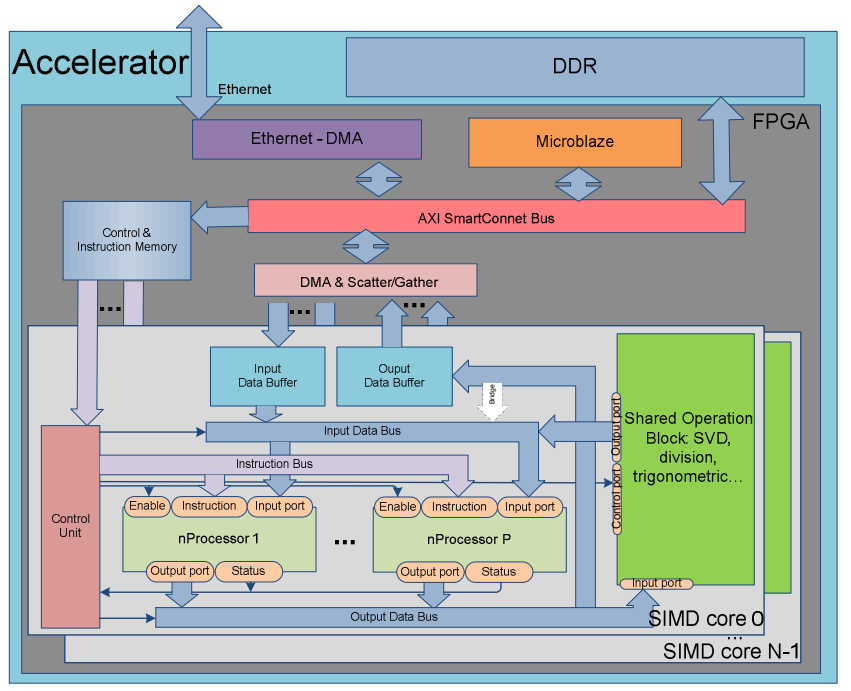

Figure 1: The FPGA-based accelerator architecture 
Additionally, Figure 1 shows the extra control and communication elements which are needed. the AXI Smart Connect Bus, or simply AXI, is the central block that communicates all the elements within the accelerator. Microblaze is an embedded general-purpose processor that acts as the master in the architecture and it is in charge of controlling and configuring the architecture. It manages the internal data flows, distributes the input data between the SIMD processing cores, and organizes the output of the results. The communications are based on Ethernet. This lets our accelerator to work in a stand-alone manner. The Ethernet-DMA block manages the communication flows according to the Microblaze commands. The DDR works as an input and output buffer over the data flows. The input data are stored in the external DDR memory for waiting to be processed by the SIMD cores and the results are stored in the DDR before being sent back by Ethernet. SIMD architectures usually use only one instruction memory since there is only one instruction stream. We use this memory also for storing some control instructions, as reflected by Control \& Instruction Memory (CIM) in Figure 1. In order to save memory resources, we have extracted the CIM from each original SIMD core and only one CIM distributes the instructions to the different SIMD cores. The TAPAS software tool [1] automates the entire design process and system settings from an input algorithm code. This tool uses advanced techniques of software pipelining and parallelizing scientific algorithms in multicore systems.

\section{A prototype for high-performance computing}

The immediate application of our accelerator is for executing the same scientific solar physics algorithm as for the original proposal aboard Solar Orbiter. Taken our proposal of a generic accelerator presented above and the new Xilinx XCVU9P FPGA we propose a multi-core system-on-chip, embedded on FPGA, for (close to) real-time data processing to be used with the Daniel K. Inouye Solar Telescope (DKIST). Our system will carry out a complex solar physics program that will provide "quick-look" maps of the vector magnetic field and the line-of-sight (LOS) velocity of the solar plasma. Our proposal is to use a stand-alone FPGA-based device capable of carrying out a very fast scientific analysis and the name of our prototype is INFACT from "inversion factory".

INFACT will be allocated in the Ethernet network within the DKIST Data Center [2]. It admits up to Gigabit Ethernet. This organization lets to any final user within the network to invoke the inversion since it is another computing element like supercomputers or servers where the entire data processing pipeline is executed. In fact, thanks to a software library it is integrated like an auxiliary software function within the mentioned pipeline.

The accelerator architecture is composed by 40 SIMD cores. This model roughly supposes 480 processors and 40 SOB cores working in parallel to attack the scientific problem. In addition, since each processor is managing two threads, in a given time INFACT processes up to 960 threads in parallel.
It is very important to remark that since our prototype is integrated in the local network, its performance is very scalable because several devices could be allocated in such network. In addition, the software library supports this kind of upgrade because the IP address is configurable by the software in runtime. The final stand-alone device will be integrated in the processing data pipeline and it is competitive in computing speed to computer clusters since we can replicate within the Xilinx XCVU9P FPGA up to 50 times the previous design.

\section{Conclusions}

We have presented a scientific computing accelerator on FPGA that can be used as a computing server on a network. It is based on several SIMD cores working in parallel. A prototype of our proposed accelerator, called INFACT, will be integrated within the DKIST Data Center local network as a server to analyze solar images for DKIST. The processing architecture embedded on FPGA is an evolution of the computing proposal for the SO/PHI space instrument. Basically, the architecture is composed by 40 SIMD cores. This model roughly includes 480 processors and 40 SVD cores working in parallel. In addition, since each processor is managing two threads, in a given time INFACT processes up to 960 threads in parallel. We have presented a device 40 times more powerful than the one used by SO/PHI. Taking into account that the latter is faster than a desktop computer in a factor up to 10 times, as shown in [1], then we can expect INFACT to provide a performance very competitive with a cluster composed by 50 processors using a single FPGA device.

We have presented the internal accelerator architecture based on several SIMD cores linked through a generic AXI bus. The generation and configuration of the architecture is automatized using the TAPAS software tool which will also permit to reconfigure the FPGA for carrying out different scientific algorithms. The accelerator is focused in scalability, adaptability to different FPGA devices, and to different and generic scientific algorithms.

\section{ACKNOWLEDGMENTS}

This work has been partially funded by the Spanish Ministerio de Ciencia, Innovación y Universidades, through Projects No.

ESP2016-77548-C5-1-R and RTI2018-096886-B-C51, including a percentage from European FEDER funds. Authors also acknowledge financial support from the State Agency for Research of the Spanish MCIU through the "Center of Excellence Severo Ochoa" award to the Instituto de Astrofísica de Andalucía (SEV-2017-0709)

\section{REFERENCES}

[1] Cobos Carrascosa, J.P.; Ramos, J.L.; Aparicio del Moral, B.; Balaguer M.; Lopez Jimenez, A.C.; del Toro Iniesta, J.C. "SIMD architecture on FPGA for scientific computing aboard a space instrument". Journal of Systems Architecture. Volume 62, January 2016, Pages 1-11, ISSN 1383-7621

[2] S. Berukoff, T. Hays, K. Reardon, DJ Spiess, F. Watson, S. Wiant, "Petascale cyber infrastructure for ground-based solar physics: approach of the DKIST data center," Proc. SPIE 9913, Software and Cyber infrastructure for Astronomy IV, 99131F (26 July 2016); doi: 10.1117/12.2231899 